\section{Experimental \\ demonstration of an \\ ultracompact polarization beamsplitter based on a multimode interference coupler with internal photonic crystals}

\author{
Yaocheng Shi, ${ }^{a, b}$ Naeem Shahid, ${ }^{b}$ Mingyu Li, ${ }^{a, b}$ \\ Audrey Berrier, ${ }^{\text {b, }}$ Sailing He, ${ }^{\text {a,b }}$ FELLOW SPIE, and \\ Srinivasan Anand ${ }^{b}$ \\ ${ }^{a}$ Zhejiang University, Joint Research Center of Photonics \\ of the Royal Institute of Technology, Sweden, and \\ Zhejiang University, Hangzhou 310058, China \\ ${ }^{\mathrm{b}}$ Royal institute of Technology, School of Information and \\ Communication Technology, Electrum 229, S16440 \\ Kista, Sweden \\ E-mail: anand@kth.se
}

\begin{abstract}
The fabrication and characterization of a compact InP-based polarization beamsplitter (PBS) is presented. A multimode interference (MMI) coupler with an internal air hole photonic crystal (PhC) section is utilized to separate the two polarizations. The PhC structure in the middle of the $\mathrm{MMI}$ is polarization dependent, so that one polarization is reflected and the other one is transmitted; both are collected by the respective output ports of the MMI coupler. The obtained experimental results show that the PBS as short as $\sim 400 \mu \mathrm{m}$ has an extinction ratio as large as $15 \mathrm{~dB}$. (๑) 2010 Society of Photo-Optical Instrumentation Engineers.

[DOI: $10.1117 / 1.3454377]$
\end{abstract}

Subject terms: polarization beamsplitter; multimode interference; photonic crystal; InP.

Paper 100177LR received Mar. 5, 2010; revised manuscript received May 11, 2010; accepted for publication May 19, 2010; published online Jun. 28, 2010.

\section{Introduction}

Polarization beamsplitters (PBSs) utilized to split or combine beams with two orthogonal polarization states are widely applied in optical communications, sensing, data storage, and imaging. ${ }^{1,2}$ Various types of PBS have been reported on silica, $\mathrm{LiNbO}_{3}$, InP, and also polymer materials. Material birefringence or stress-induced birefringence has been used to realize polarization splitting/combining. ${ }^{3,4}$ Multimode interference (MMI) couplers ${ }^{5}$ have also been used for the realization of polarization splitting. However, PBSs based on conventional MMI usually have a large size, since the total length should be integer multiples of the coupling lengths for both polarizations.

Photonic crystals (PhCs) have attractive spectral and polarization-dependent properties suitable for photonic de-

\footnotetext{
* Current affiliation: FOM Institute for Atomic and Molecular Physics (AMOLF), Center for Nanophotonics c/o Philips Research, 5656AE Eindhoven, The Netherlands.
}

0091-3286/2010/\$25.00 @ 2010 SPIE vices. Different schemes have been reported to use $\mathrm{PhC}$ structures, primarily 2-D PhCs, to split different polarization states. PBS based on PhC that utilize different band structures for two polarizations or anisotropy for two polarizations has been reported. ${ }^{6-8}$ PBS in InP-based materials are attractive for integrated components based on the InPtechnology platform, which is a natural choice for optical communication wavelengths, since it provides both passive and active functionality. Further, it is interesting to investigate new device concepts that combine conventional components with PhCs. In this work, we present an InP-based ultracompact PBS realized by combining a MMI coupler with $\mathrm{PhC}$. It is well known that $\mathrm{PhCs}$ have a polarizationdependent response due to the inherent anisotropy of the periodic structure. By carefully choosing the lattice parameters of the $\mathrm{PhC}$ for the desired wavelength of operation, one can obtain a PhC that will have a bandgap for only one polarization while it is absent for the other. By suitably placing such a $\mathrm{PhC}$ structure in the multimode section of the MMI coupler, one polarization can be reflected, and the other one transmitted through and collected by the respective output ports of MMI. In this work, we focus on the experimental validation of the principle of operation of the PBS. The obtained measurement results show qualitative agreement with the simulation results.

\section{Design and Analysis}

The schematic configuration of the PBS based on MMI with internal $\mathrm{PhC}$ is shown in Fig. 1. It consists of one input port (port 1), two output ports (port 2 and port 3), a multimode region, and a $\mathrm{PhC}$ structure located in the middle of the multimode region. For ease of access in measurements, a bent waveguide section is used so that both the output ports are on the same side of the device.

The operation of the MMI coupler here is similar to that of a regular MMI splitter, while the internal $\mathrm{PhC}$ structure works as a polarization sensitive reflector. By assuming that transmission $(t)$ and reflection $(r)$ coefficients for all the guided modes are the same, the forward propagating field $\psi_{t}\left(x, L_{t}\right)$ and the backward propagating field $\psi_{r}(x, 0)$ are given by, ${ }^{9}$

$\psi_{t}\left(x, L_{t}\right)=t \cdot \sum_{\nu} c_{\nu} \varphi_{\nu}(x) \exp \left[j \frac{\nu(\nu+2) \pi}{3 L_{\pi}} L_{t}\right]$,

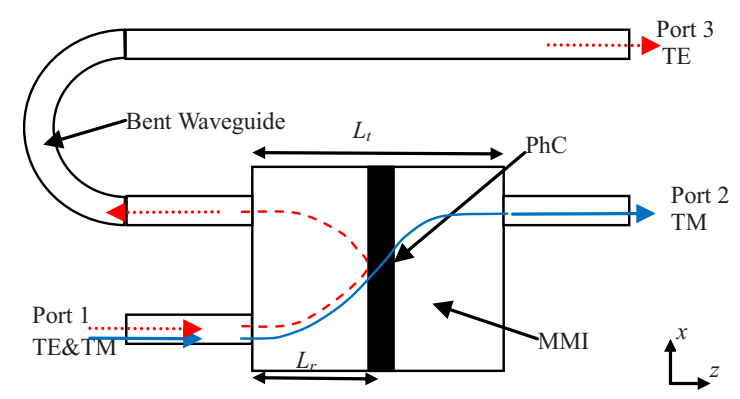

Fig. 1 Schematic illustration of the PBS with the input and output waveguides. 

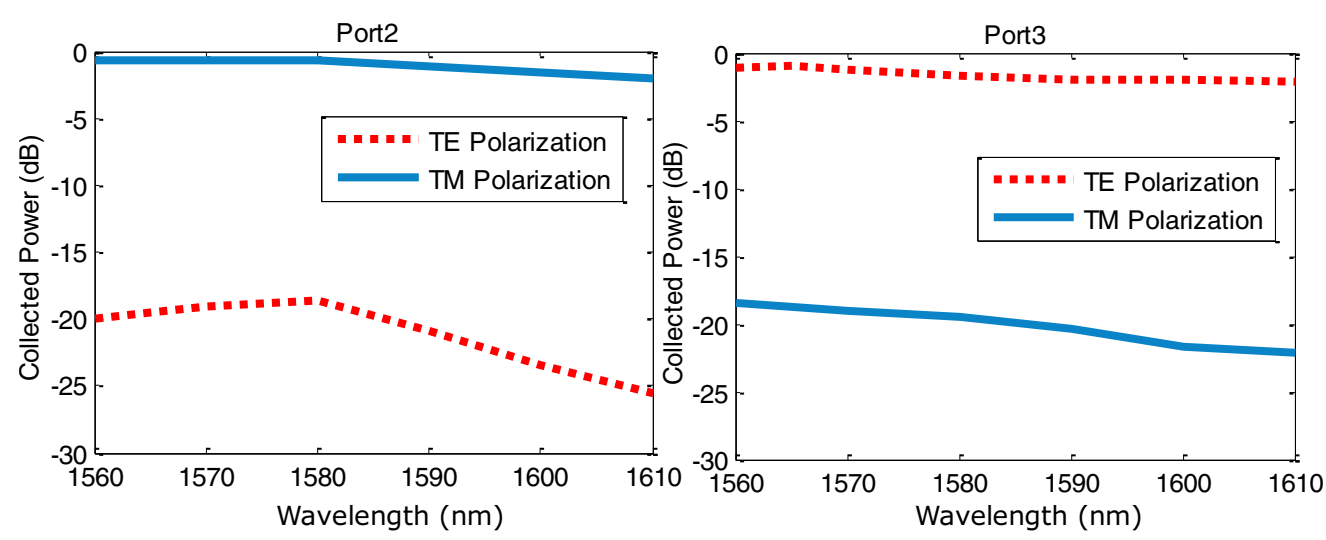

Fig. 2 Optical power spectra at the two output ports of the PBS obtained by 2-D FDTD simulations.

$\psi_{r}(x, 0)=r \cdot \sum_{\nu} c_{\nu} \varphi_{\nu}(x) \exp \left[j \frac{\nu(\nu+2) \pi}{3 L_{\pi}} \cdot 2 \cdot L_{r}\right]$,

where $c_{\nu}$ and $\varphi_{\nu}(x)$ are the excitation coefficient and modal field of the $\nu^{\prime}$ th order mode, respectively; $L_{r}$ is the distance between the beginning (input edge) of the MMI and the $\mathrm{PhC}$; and $L_{t}$ is total length of the MMI section.

By setting $L_{t}=L_{\pi}^{\mathrm{TM}}$ and $L_{r}=L_{\pi}^{\mathrm{TE}} / 2$, we obtain

$\psi_{t}\left(x, L_{t}\right)=t \cdot \psi(-x, 0)$,

$\psi_{r}(x, 0)=r \cdot \psi(-x, 0)$.

One can find that single-fold self-images are formed in the forward and backward directions if appropriate lengths $L_{t}$ and $L_{r}$ are chosen. The image quality also depends on the transmission and reflection coefficients $t$ and $r$, respectively. The PhC structure should be designed to make the TE and TM polarizations highly reflected and transmitted, respectively.

In this work, we consider a $\mathrm{PhC}$ structure with a triangular lattice of air holes (with period $a$ and hole radius $R$ ) in an $\mathrm{InP} / \mathrm{GaInAsP} / \mathrm{InP}$ heterostructure [with refractive indices $n=3.17$ (InP), 3.35 (GaInAsP)]. The upper InP cladding is $200 \mathrm{~nm}$ thick and the GaInAsP core layer is $420 \mathrm{~nm}$. The 2-D simulations of the PhC were performed using the effective index approximation. For the slab structure used in this work, the effective index is $n_{\mathrm{eff}}=3.24$. For $0.2<R / a<0.43$, only TE polarization has a bandgap, while it is absent for TM polarization. Such PhC structures could be used to separate the two polarizations. One may comment that small $R / a$ is preferred, since the optical losses in the $\mathrm{PhC}$ increase with $R / a$. But at the same time, the bandgap should be large enough to ensure a large bandwidth. Finally, as a tradeoff between these two requirements, the radius of the air hole is chosen to be $R=0.26 a$. In the near-infrared region of interest, only TE polarization has a bandgap ranging from 0.215 to $0.256(a / \lambda)$; however, no bandgap is observed for the TM polarization. In this case, the transmission and reflection coefficients of such a $\mathrm{PhC}$ structure for TE and TM polarizations should be $t_{\mathrm{TE}}$ $\approx 0, r_{\mathrm{TE}} \approx 1$ and $t_{\mathrm{TM}} \approx 1, r_{\mathrm{TM}} \approx 0$.

The width of the multimode section is chosen to be $6 \mu \mathrm{m}$ to ensure high quality self-images. The optimized length for the MMI section is $98 \mu \mathrm{m}$ and the $\mathrm{PhC}$ section is placed at $L_{r}=48.5 \mu \mathrm{m}$. The length of the $\mathrm{PhC}$ is chosen to be 11 periods (14 rows in the $\Gamma \mathrm{M}$ direction) to ensure large enough reflection for TE polarization. We use a 2-D finitedifference time-domain (FDTD) method with perfectly matched layer (PML) boundary treatment for numerical simulation. The grid sizes are chosen to be $\Delta x=\Delta z$ $=10 \mathrm{~nm}$ for achieving convergent simulation results. The wavelength response of the device is given in Fig. 2, and the results show that the proposed PBS has very good extinction ratios over the full wavelength range.

\section{Fabrication and Characterization}

The InP/InGaAsP/InP heterostructure corresponding to the parameters used in the simulations was grown on an InP substrate by metal organic vapor phase epitaxy (MOVPE). The InP upper cladding layer is $200 \mathrm{~nm}$ thick, while the InGaAsP core layer $\left(\lambda_{\text {gap }}=1.22 \mu \mathrm{m}\right.$, lattice matched to InP) is $420 \mathrm{~nm}$ thick. A 260-nm-thick $\mathrm{SiO}_{2}$ layer (etch mask) was deposited on top of the InP layer. Using ZEP as the resist, the PBS pattern was defined using e-beam lithography (Raith 150) with an acceleration voltage of $25 \mathrm{kV}$. The pattern was transferred into $\mathrm{SiO}_{2}$ mask by fluorine-based reactive ion etching. Subsequently, the sample was etched using $\mathrm{Ar} / \mathrm{Cl}_{2}$ chemically assisted ion beam etching (CAIBE). Figure 3 shows the SEM pictures of the fabricated PBS; the top view of the MMI region and the cross section showing the deeply etched $\mathrm{PhC}$ are also shown. A bent waveguide with a bending radius $R_{0}=100 \mu \mathrm{m}$ is placed at the reflection port to ensure both outputs at the

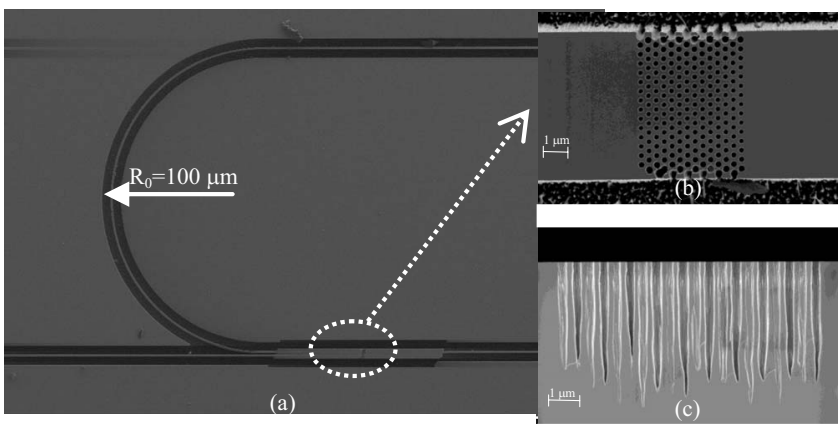

Fig. 3 SEM pictures for the fabricated PBS: (a) top view, (b) internal $\mathrm{PhC}$, and (c) cross section of the etched PhC. 

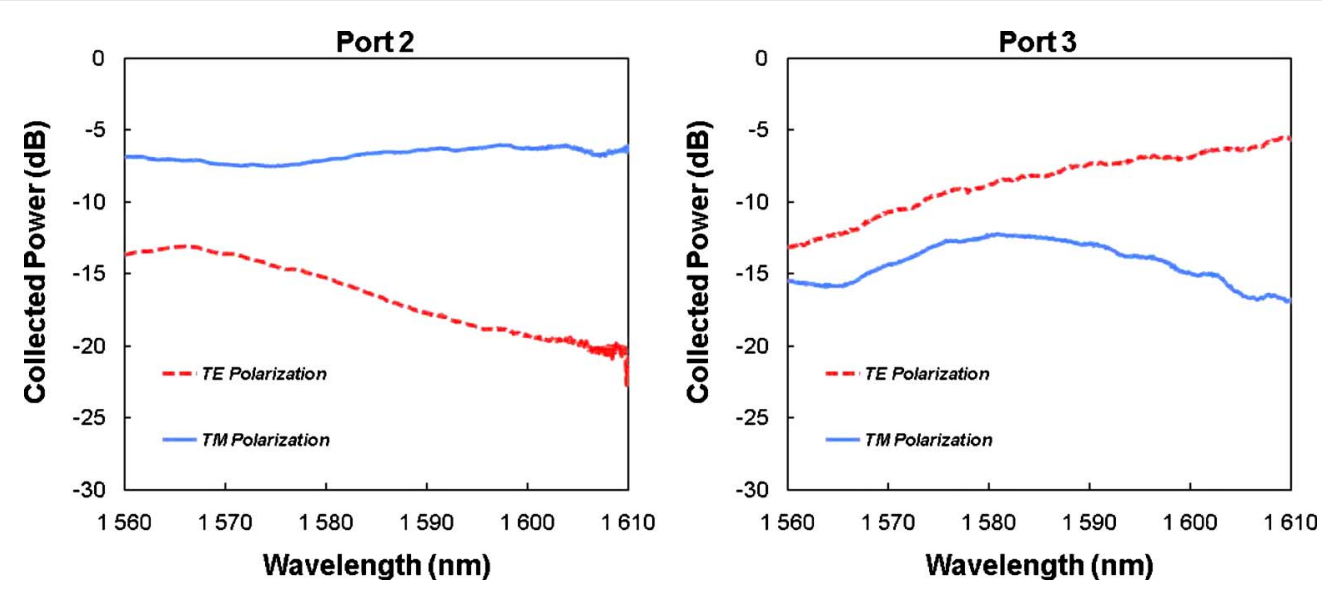

Fig. 4 Measured optical power spectra for the two output ports of the PBS.

same facet. The total size of the device is about 400 $\times 200 \mu \mathrm{m}^{2}$ (including the access ridge waveguides), which is quite compact compared to those based on conventional designs.

The end-fire method was used to optically characterize the fabricated PBS. We use an amplified spontaneous emission (ASE) source with a broadband spectrum range of 1560 to $1610 \mathrm{~nm}$ to characterize the device. Measured transmission spectra of the two output ports for TE and TM polarizations are shown in Fig. 4.

In the wavelength range from 1590 to $1610 \mathrm{~nm}$, the measurements show an extinction ratio in the range 10 to $15 \mathrm{~dB}$ for the transmission port (port 2), while a somewhat lower value $(5$ to $10 \mathrm{~dB})$ is observed for the reflection port (port 3 ). The measurements are qualitatively consistent with the simulation results (Fig. 2) and demonstrate experimental validation of the PBS. However, quantitatively the experimental results show some deviations with respect to the simulations. This discrepancy is due to the 2-D approximation used in the simulations. Since the problem is inherently $3-\mathrm{D}$, the geometrical parameters $(\mathrm{PhC}$ location and dimensions of the MMI section) used in the 2-D approximation (design and simulations) may not represent optimized values for the device. Nevertheless, although qualitative, the 2-D simulations give a good representation of the functioning of the device. Our future work will investigate improvements in fabrication, together with optimization of the geometrical parameters of the device using 3-D simulations to obtain better performances.

\section{Conclusion}

A PBS based on a MMI coupler with an internal $\mathrm{PhC}$ is fabricated and characterized. The fabricated device is ultra- compact, having a size of $400 \times 200 \mu \mathrm{m}^{2}$, and is compatible with integration on InP. The results show that polarization-dependent transmission/reflection properties of an internal $\mathrm{PhC}$ mirror can be used to separate the two polarization states.

\section{Acknowledgments}

This work was supported by the Swedish Research Council and the Swedish Strategic Research Foundation.

\section{References}

1. C. Tai, S. Chang, and T. Chiu, "Design and analysis of an ultracompact and ultra-wideband polarization beam splitter based on coupled plasmonic waveguide arrays," IEEE Photon. Technol. Lett. 19, 1448-1500 (2007).

2. Y. Shi, D. Dai, and S. He, "Proposal for an ultra-compact polarization beam splitter based on a photonic-crystal-assisted multimode interference coupler," IEEE Photon. Technol. Lett. 19, 825-827 (2007).

3. T. K. Liang and H. K. Tsang, "Integrated polarization beam splitter in high index contrast silicon-on-insulator waveguides," IEEE Photon. Technol. Lett. 17, 393-395 (2005).

4. N. Miliou, R. Srivastava, and R. V. Ramaswamy, "A 1.3- $\mu \mathrm{m}$ directional coupler polarization splitter by ion exchange," J. Lightwave Technol. 11, 220-225 (1993).

5. D. M. Mackie, T. J. Tayag, and T. E. Batchman, "Polarization separation/combination based on self-imaging," Opt. Eng. 40, 2265$2272(2001)$

6. S. Kim, G. P. Nordin, J. Cai, and J. Jiang, "Ultracompact highefficiency polarizing beam splitter with a hybrid photonic crystal and conventional waveguide structure," Opt. Lett. 28, 2384-2386 (2003).

7. V. Mocella, P. Dardano, L. Moretti, and I. Rendina, "A polarizing beam splitter using negative refraction of photonic crystals," Opt. Express 13, 7699-7707 (2005)

8. V. Zabelin, L. A. Dunbar, N. Le Thomas, R. Houdré, M. V. Kotlyar, L. O' Faolain, and T. F. Krauss, "Self-collimating photonic crystal polarization beam splitter," Opt. Lett. 32, 530-532 (2007).

9. L. W. Chung and S. L. Lee, "Multimode-interference-based broadband demultiplexers with internal photonic crystals," Opt. Express 14, 4923-4927 (2006). 